Proc. of the 13th Symposium of Magnetic Measurements and Modeling SMMM'2018, Wieliczka, Poland, October 8-10, 2018

\title{
Sputtered $\mathrm{Y}_{3} \mathrm{Fe}_{5} \mathrm{O}_{12}$ Films for Spintronics Application
}

\author{
B. Guzowski ${ }^{a, *}, \mathrm{R}$. Gozdur ${ }^{a}$ And A. Kociubiński ${ }^{b}$ \\ ${ }^{a}$ Lodz University of Technology, Department of Semiconductor and Optoelectronics Devices, \\ Wolczanska 211/215, 90-924 Lodz, Poland \\ ${ }^{b}$ Lublin University of Technology, Institute of Electronics and Information Technology, \\ Nadbystrzycka 38A, 20-618 Lublin, Poland
}

\begin{abstract}
This paper presents detailed investigation of sputtered $\mathrm{Y}_{3} \mathrm{Fe}_{5} \mathrm{O}_{12}$ (YIG) films dedicated to spintronics devices. YIG films were sputtered on ceramic and glass substrates. Magnetic properties of the developed films were analyzed and compared with magnetic properties of pure YIG crystal. Magnetic properties of YIG film sputtered on ceramic substrates are very similar to measured properties of the reference sample.
\end{abstract}

DOI: 10.12693/APhysPolA.136.693

PACS/topics: 85.80.Fi, 75.70.Ak, 75.60.Ej

\section{Introduction}

Yttrium iron garnet, $\mathrm{Y}_{3} \mathrm{Fe}_{5} \mathrm{O}_{12}$ (YIG) is a widely used ferrimagnetic insulator because of its excellent parameters such as: low microwave loss, high resistivity, and good transparency. Therefore YIG is irreplaceable in microwave [1], optoelectronics [2], magneto-optical [3], and magnetic [4] applications. Since YIG has the lowest magnon damping of all known materials [5] and because magnon propagation is observed on over centimeter distances [5], YIG has become very attractive in spintronics. YIG with nonmagnetic metal platinum $(\mathrm{Pt})$, which has a very strong spin-orbit coupling, is one of the most frequently explored bilayer in spin caloritronics $[6,7]$ beside NiFe substrates [8]. Structure YIG/Pt has been used during investigation of many pure spin current phenomena, including inverse spin Hall effect (ISHE) [9], spin Hall effect [10], spin pumping [11], or spin Seebeck effect (SSE) [7]. YIG/Pt is widely used in longitudinal SSE and it has been recently proved $[12,13]$ that SSE signal is practically not contaminated by anomalous Nernst effect (ANE) signals. However, the induced ferromagnetism in $\mathrm{Pt}$ may disturb pure spin current phenomena in YIG/Pt bilayer due to magnetic proximity effect [14]. High efficiency of spin Hall effect in YIG/Pt bilayers makes them useful for application in thermoelectric power generators.

YIG layers have been fabricated for more than forty years [15], but since YIG layers have been used in spintronic application, new methods have been developed. Nowadays YIG films are fabricated by various methods: liquid phase epitaxy (LPE) [16], RF magnetron sputtering [17], or pulsed laser deposition (PLD) [18].

Since the cost of YIG crystal is very high in comparison to NiFe substrates, one of the main objectives of the conducted research is to decrease the cost of YIG substrates

\footnotetext{
* corresponding author; e-mail bartlomiej.guzowski@p.lodz.pl
}

and to improve the magnetic properties of these substrates. If the price of YIG/Pt bilayers is reduced, they can be widely used in thermoelectric applications. In this paper low cost fabrications process of YIG layers for spintronic applications is given. Detailed investigation of the sputtered layers is presented.

\section{Sample preparation}

In the conducted research the RF magnetron sputtering with argon plasma method has been used. This method allows to fabricate sub- $\mu$ m-thick layer, which are very useful for spin caloritronics applications. The thin films of YIG were sputtered by deposition system Nano 36 from Kurt J-Lesker. Time of deposition was $8 \mathrm{~h}$ for $200 \mathrm{~nm}$ films and $4.5 \mathrm{~h}$ for $100 \mathrm{~nm}$ films. Argon flow rates was $130 \mathrm{sccm}$, sputtering power was $250 \mathrm{~W}$, and operating pressure was from 1 to 50 mTorr. During the research three types of samples were fabricated, as shown in Fig. 1. In sample 1 (Fig. 1a) $200 \mathrm{~nm}$ thick YIG film was sputtered on a glass substrate. In sample 2 (Fig. 1b) the substrate was made of $\mathrm{Al}_{2} \mathrm{O}_{3}$ and the thickness of the sputtered YIG film was $100 \mathrm{~nm}$. In sample 3 (Fig. 1c) $100 \mathrm{~nm}$ thick YIG film was sputtered on a glass substrate.

\section{(b)}

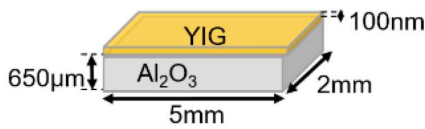

(a)

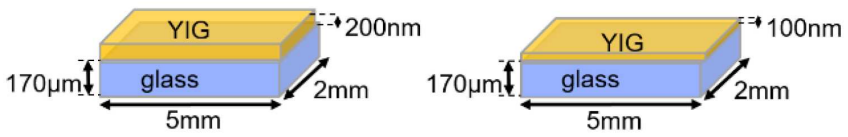

Fig. 1. Sample 1: $200 \mathrm{~nm}$ YIG on glass (a), sample 2: $100 \mathrm{~nm}$ YIG on $\mathrm{Al}_{2} \mathrm{O}_{3}$ (b), sample 3: $100 \mathrm{~nm}$ YIG on glass (c). 


\section{Results and discussion}

The developed substrates were characterized by EDS probe X-MAX N80 from Oxford Instruments and scanning electron microscope (SEM). Photos taken by SEM of YIG films sputtered on sample 1 is shown in Fig. 2. As a reference sample a piece of pure YIG crystal was used.

The photo of a reference sample surface is shown in Fig. 3a, while the surface of YIG sample sputtered on ceramic substrate is given in Fig. 3b. The measurements and calculations of percentage weights of YIG films are collected in Table I. EDS spectrum of reference for the reference sample and sputtered YIG film is shown in Fig. 4. The magnetic properties of the sputtered YIG films were recorded by VersaLab System (Quantum Design). Another method which allows measurement of magnetic parameter of magnetic materials has a shunt resistor and pick-up coils [19] and is compatible with the recommendations of IEC 60404 standards.

Measured results for YIG thin films are shown in Fig. 5a while in Fig. 5b reference hysteresis loop measured for pure YIG target is given.

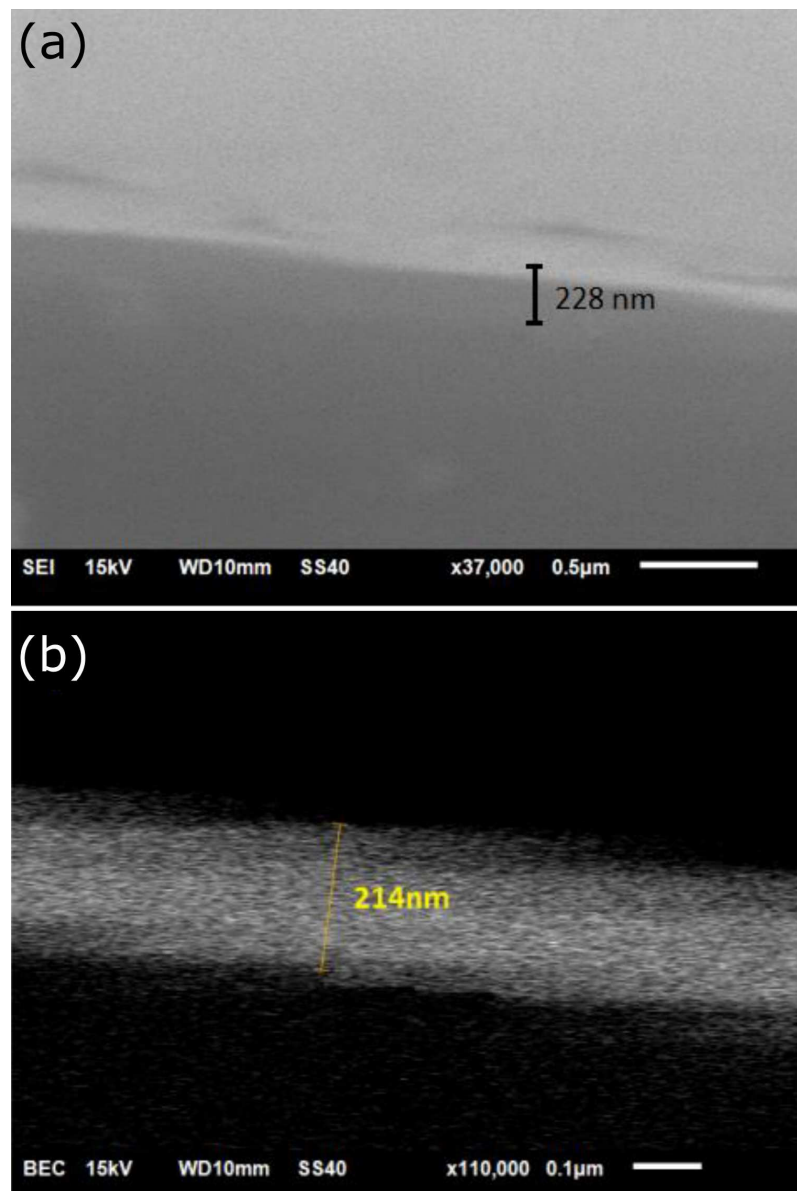

Fig. 2. Photo of YIG films on sample 1 taken by scanning electron microscope with magnification $37,000 \times$ (a) and $110,000 \times(b)$
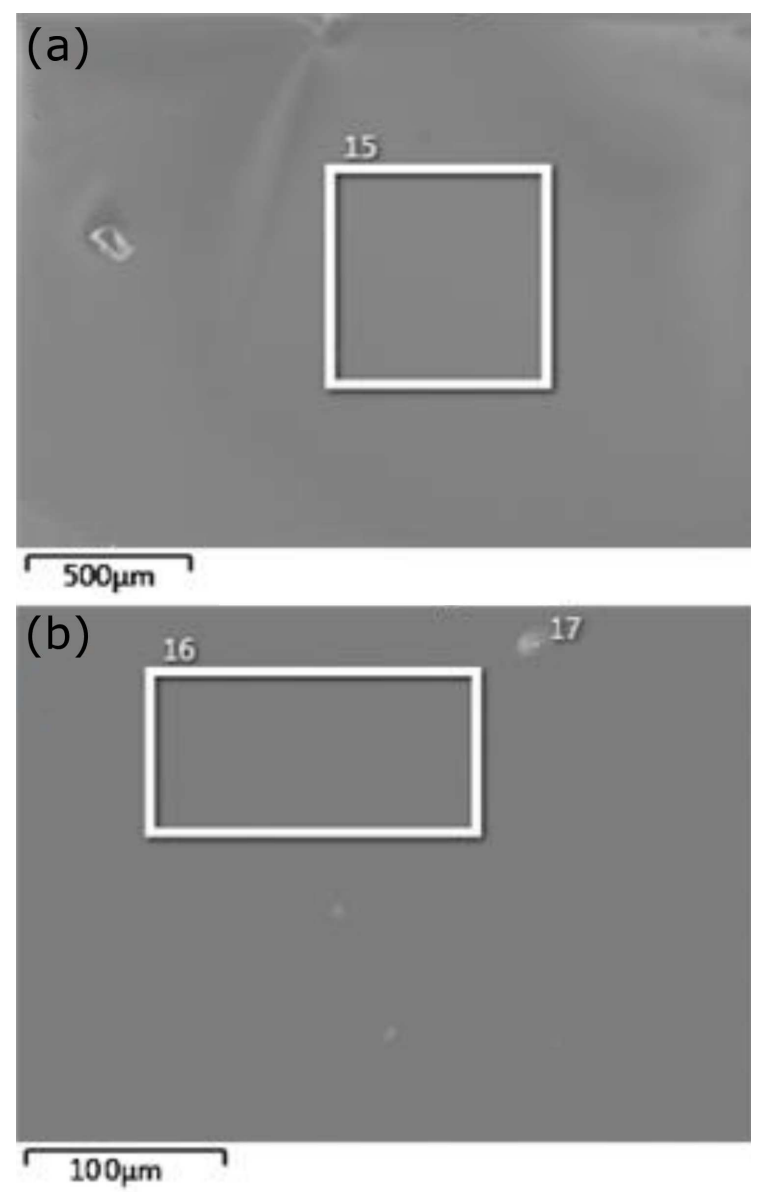

Fig. 3. Surface of reference sample (a), surface of YIG film on sample 2 (b).
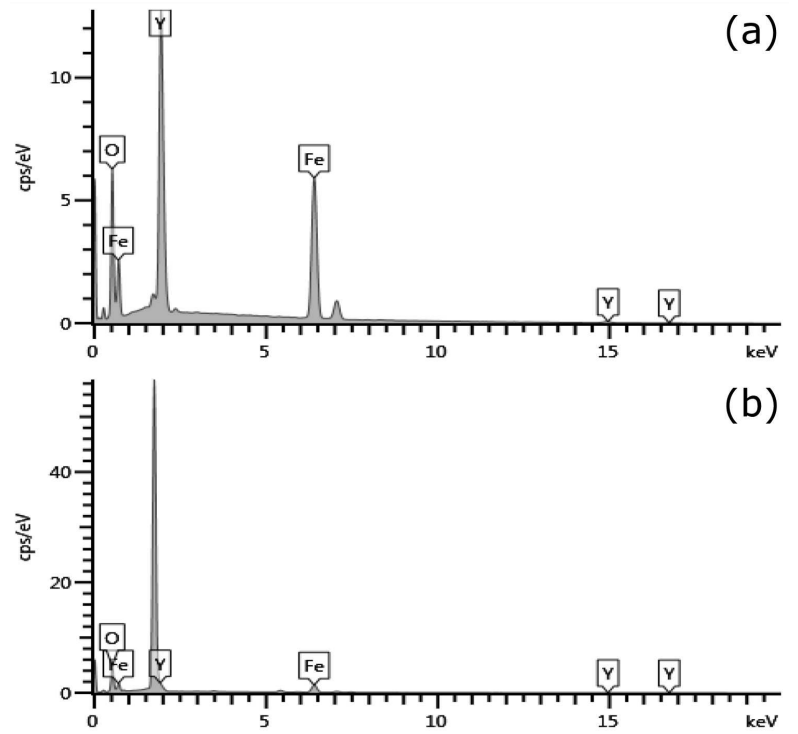

Fig. 4. EDS spectrum of reference sample (a), EDS spectrum of YIG film on sample 2 (b). 
TABLE I

The nominal and the measured YIG composition (wt\%)

\begin{tabular}{c|c|c|c}
\hline \hline Element & $\mathrm{O}$ & $\mathrm{Fe}$ & $\mathrm{Y}$ \\
\hline nominal & 26.75 & 24.56 & 38.7 \\
measured & 28.38 & 56.82 & 14.8
\end{tabular}
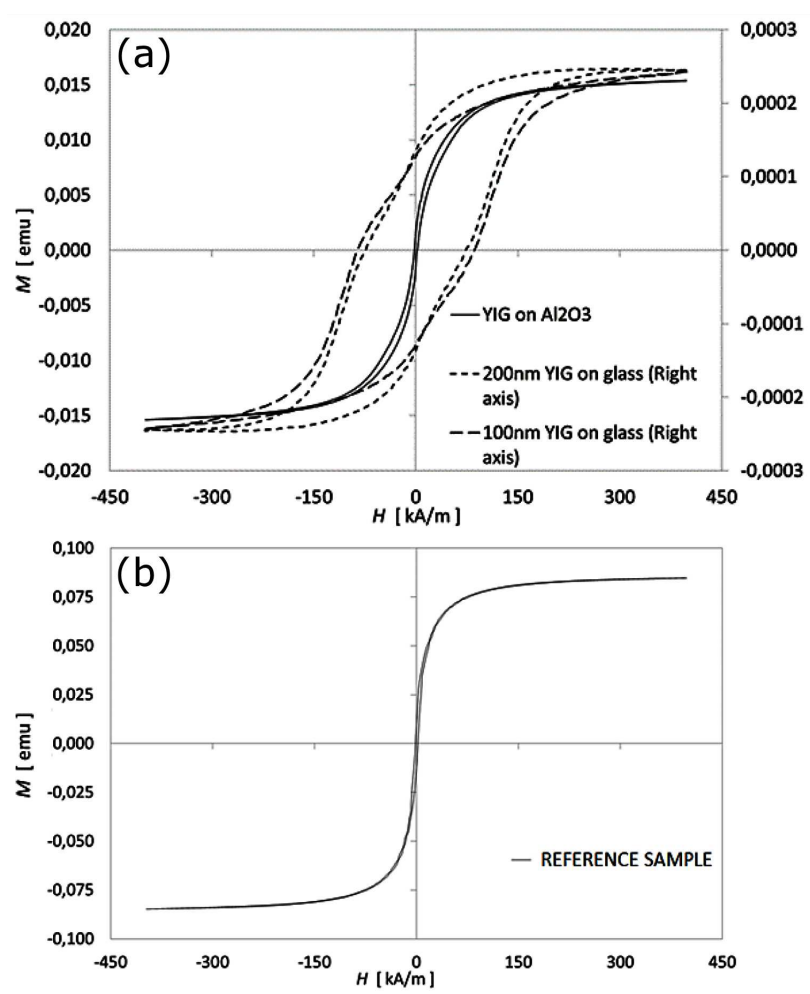

Fig. 5. Set of hysteresis loops measured for developed YIG films (a), measured hysteresis loop of reference sample (b).

The highest convergence of measurements with the reference sample was observed for sample 2. Sample 1 and sample 3 have very poor magnetic properties (Fig. 5a). Deterioration of magnetic parameters of these samples may be related to the preparation of test samples or a local change of magnetic parameters. Sample 1 and sample 3 were rejected for further investigation.

\section{Conclusions}

In this paper magnetic properties of thin YIG films were fabricated with magnetron sputtering process. YIG films were deposited on glass and $\mathrm{Al}_{2} \mathrm{O}_{3}$ in the same technological process. On the basis of the obtained results it can be concluded that YIG films on glass have much worse magnetic properties in comparison to YIG films deposited on $\mathrm{Al}_{2} \mathrm{O}_{3}$. YIG films sputtered on ceramic have very similar magnetic properties to the measured properties of the reference sample. The smallest coercive field $\boldsymbol{H}_{c}$ was measured for sample $2 \boldsymbol{H}_{c}=2.5 \mathrm{kA} / \mathrm{m}$ and was the closest to nominal parameter. The worst parameters were measured for sample 3 with $\boldsymbol{H}_{c}=86.7 \mathrm{kA} / \mathrm{m}$ and sample 1 with $\boldsymbol{H}_{c}=73.5 \mathrm{kA} / \mathrm{m}$. It could be caused by the presence of structural defects in the magnetic film sputtered on the glass substrate. Magnetron sputtering seems to be a low cost method to fabricate the YIG substrate for spintronic devices, however further research must be carried out to improve the composition of deposited films.

\section{References}

[1] V. Sharma, J. Saha, S. Patnaik, B.K. Kuanr, J. Magn. Magn. Mater. 439, 277 (2017).

[2] S. Ghosh, S. Keyvavinia, W. Van Roy, T. Mizumoto, G. Roelkens, R. Baets, Opt. Exp. 20, 1839 (2012).

[3] T. Boudiar, B. Payet-Gervy, M.F. Blanc-Mignon, J.J. Rousseau, M.L. Berre, H. Joisten, J. Magn. Magn. Mater. 284, 77 (2004).

[4] A.K. Bandyopadhyay, S.E. Rios, S. Fritz, J. Garcia, J. Contreras, C.J. Gutierrez, Trans. Magn. 40, 2805 (2004).

[5] A.J. Princep, R.A. Ewings, S. Ward, S. Tóth, C. Dubs, D. Prabhakaran, A.T. Boothroyd, NPJ Quant. Mater. 2, 1 (2017).

[6] K. Uchida, J. Xiao, H. Adachi, J. Ohe, S. Takahashi, J. Ieda, T. Ota, Y. Kajiwara, H. Umezawa, H. Kawai, G.E.W. Bauer, S. Maekawa, E. Saitoh, Nat. Mater. 9, 894 (2010).

[7] K. Uchida, H. Adachi, T. Ota, H. Nakayama, S. Maekawa, E. Saitoh, Appl. Phys. Lett. 97, 172505 (2010).

[8] B. Guzowski, R. Gozdur, M. Lakomski, Acta Phys. Pol. A 133, 541 (2018).

[9] C. Hahn, G.D. Loubens, O. Klein, M. Viret, V.V. Naletov, J.B. Youssef, Phys. Rev. B 87, 174417 (2013).

[10] Y. Kajiwara, K. Harii, S. Takahashi, J. Ohe, K. Uchida, M. Mizuguchi, H. Umezawa, H. Kawai, K. Ando, K. Takanashi, S. Maekawa, E. Saitoh, $\mathrm{Na}$ ture 464, 262 (2010).

[11] S. Emori, A. Matyushov, H.M. Jeon, C.J. Babroski, T. Nan, A.M. Belkessam, J.G. Jones, M.E. McConney, G.J. Brown, B.M. Howe, N.X. Sun, Appl. Phys. Lett. 112, 182406 (2018).

[12] B.F. Miao, S.Y. Huang, D. Qu, C.L. Chien, AIP Adv. 6, 015018 (2016).

[13] S.R. Boona, R.C. Myers, J.P. Heremans, En. Envir. Sci. 7, 885 (2014).

[14] S.Y. Huang, X. Fan, D. Qu, Y.P. Chen, W.G. Wang, J. Wu, T.Y. Chen, J.Q. Xiao, C.L. Chien, Phys. Rev. Lett. 109, 107204 (2012).

[15] A. Braginski, Trans. Magn. 7, 467 (1971).

[16] S.L. Blank, J.W. Nielsen, J. Cryst. Growth 17, 302 (1972).

[17] B. Stadler, A. Gopinath, Trans. Magn. 36, 3957 (2000).

[18] R. Karim, S.A. Oliver, C. Vittoria, Trans. Magn. 31, 3485 (1995).

[19] A. Majocha, R. Gozdur, Przegl. Elektrotechn. 86, 79 (2010)(in Polish). 